\title{
Impact of pixel-dose optimization on pattern fidelity for helium ion beam lithography on EUV resist
}

\author{
Nima Kalhor ${ }^{\mathrm{a}}$, Wouter Mulckhuyse ${ }^{\mathrm{b}}$, Paul Alkemade ${ }^{\mathrm{a}}$ and Diederik Maas* ${ }^{\mathrm{b}}$
}

${ }^{a}$ Kavli Institute of Nanoscience, Delft University of Technology, Lorentzweg 1, Delft, The

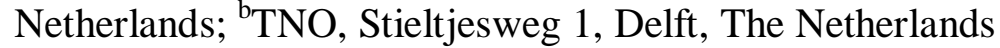

\begin{abstract}
This paper presents a heuristic model for scanning helium ion beam lithography (SHIBL) in a EUV chemically amplified resist. The model employs a point-spread function to account for all physical and chemical phenomena involved in the resist activation. Ion shot noise effects are accounted for using Poisson statistics. Our model shows a good agreement with earlier single-pixel SHIBL experiments for determining line width as a function of dose for a desired line-and-space pattern. Furthermore, we propose optimized-pixel-dose SHIBL to improve exposure latitude, LCDU and LWR. Dose optimization is advantageous to single-pixel exposure when the feature size is at least about twice the width of the FWHM of the point-spread function. We confirm this by comparing our modeling results for single-pixel and optimizedpixel-dose exposure modes for line-and-space patterns.
\end{abstract}

Keywords: Scanning helium ion beam lithography, EUV lithography, Chemically amplified resist, Metrology, Dose optimization modeling, Ion shot noise, Critical dimension, Line-width roughness

\section{INTRODUCTION}

Focused ion beam lithography (FIBL) has been investigated extensively as an alternative to e-beam lithography (EBL) for device prototyping and R\&D purposes. ${ }^{1-5}$ The studies found that FIBL offers a higher sensitivity, due to increased secondary electron (SE) yields, and a lower proximity effect, due to the absence of backscattered primary particles, compared to EBL. The development of the gas field ion source for the Zeiss Orion helium ion microscope (HIM) enables a focused ion beam with a spot-size in the sub-nanometer range, comparable or better than that which can be achieved in EBL.

Recently it was reported that there are remarkable similarities in the activation response of resists to He-ions and extreme-ultraviolet (EUV) photons in EUVL lithography (EUVL). That is, both primary particle beams result in very low proximity effects and produce low energy SEs. ${ }^{6}$ Based on this, Maas et al. demonstrated experimentally that SHIBL is indeed a promising and a cost-effective method for pre-screening chemically amplified resists (CARs), and so determining the Z-factor, ${ }^{7}$ prior to their final performance evaluation in an EUV scanner. ${ }^{6}$ However, in that work, singlepixel SHIBL in CAR resulted in poorer local critical dimension uniformity (LCDU) and slightly lower line-width roughness (LWR) to that of obtained with EUVL. This was partly attributed to the high SE yield in SHIBL which makes He-ions 150 times more effective than EUV photons in patterning a similar CAR. ${ }^{6}$ Therefore, ion shot noise may be a limiting factor in SHIBL for patterning sensitive CARs, thus hindering pattern fidelity.

In this work, we present a heuristic model for SHIBL in a CAR. We have developed a simulation model to study singlepixel SHIBL for contact hole $(\mathrm{CH})$ and line-and-space (LS) patterns. This is achieved by estimating a point-spread function (PSF) to account for all contributing factors in resist activation. We employed Poisson statistics to investigate the extent of the ion shot noise contribution on LCDU and LWR for single-pixel SHIBL. Our simulation results are in good agreement with experiments reported by Maas et al.. ${ }^{6}$ Furthermore, we investigate the advantages of optimizedpixel-dose SHIBL to improve LCDU, LWR, exposure latitude (EL) and throughput. Finally, we compare preliminary modeling results for single-pixel and optimized-pixel-dose SHIBL for a 50-nm-full-pitch LS pattern.

*diederik.maas@tno.nl; phone +31 888666 524; www.tno.nl 


\section{MODELING RESIST ACTIVATION IN CAR FOR SHIBL}

Resist activation is a complex process. Many factors (e.g. beam profile, mean free path of SEs, resist granularity, etc.) can contribute to the shape and size of the final resolved resist pattern. ${ }^{8}$ Furthermore, direct measurement of contribution by every individual factor is a significant experimental challenge.

The point-spread function (PSF), as it is considered in this paper, is the spatial distribution of the activation of a resist caused by single primary particle. A PSF can be measured by imaging single-pixel structures (e.g. dots, lines, etc.) exposed at different doses after resist development. Hence, the PSF can provide a decent estimation of the contributions of all physical and chemical phenomena involved during the exposure and post-exposure. In fact, the full width at half maximum (FWHM) of the PSF is a good parameter to quantify the beam and resist resolution. Therefore, an accurate measurement of the PSF is fundamental to model resist activation processes.

\subsection{Point-spread function for SHIBL in CAR}

Here, we explain our approach to obtain the PSF using our previous single-pixel exposure SHIBL experiments in CAR. ${ }^{6}$ Initially, the line-spread function (LSF) $F(y)$, where $y$ is the trench half-width, was obtained from the $50 \mathrm{~nm}$-fullpitch LS pattern (Figure 1(a)). The LSF exhibits a knee at a trench half-width of $11.6 \mathrm{~nm}$ with an asymptotic power-law drop-off of $1 / y^{4.5}$. Abel inversion was then employed to transform the estimated LSF into an equivalent PSF $f(r)$, where $r$ is the radius of the exposed contact hole. ${ }^{9}$ The resultant PSF exhibits a knee at a radius of $9.4 \mathrm{~nm}$ with an asymptotic power-law drop-off of $1 / r^{4}$. Figure 1(b) shows the calculated PSF and the CH pattern experimental data. Note that the PSF FWHM is equal to the twice of the PSF knee value (i.e. $18.6 \mathrm{~nm}$ ). The flat plateau regions of the LSF and PSF represent the onset dose of SHIBL for each corresponding pattern in CAR. Both LSF and PSF exhibit powerlaw-like dependences (i.e. high contrast) ${ }^{10}$ due to high sensitivity of CAR to He-ions and spatial resolution. ${ }^{6}$

Furthermore, it is worth mentioning that it has been reported that optical critical dimension (OCD) scatterometry provides a more accurate measure of $\mathrm{CD}$, for a valid number of similar features, than CD-SEM metrology. ${ }^{11-13}$ Therefore, we translated our CD-SEM metrology data for LS and CH patterns to YieldStar metrology (ASML OCD scatterometrybased metrology) data using first order fit equations provided by ASML (Veldhoven). The YieldStar estimations (3-5 nm larger than the CD-SEM measurements) for the LS and CH patterns were then used to fit the LSF and PSF, and so calculating the resist activation map as accurately as possible.
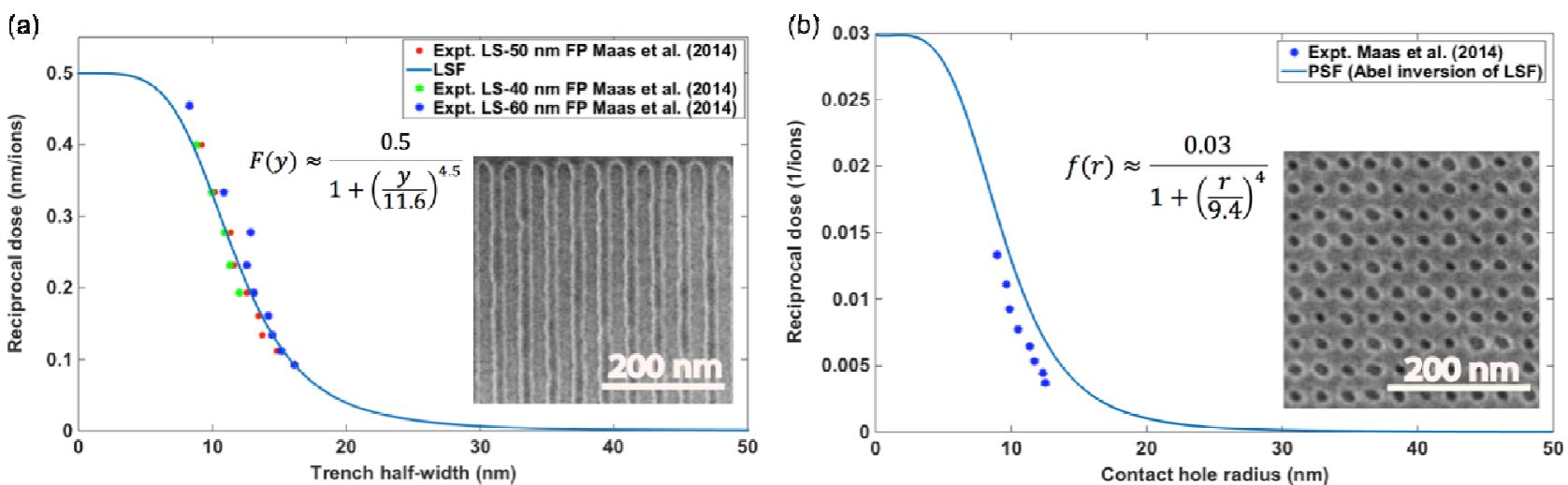

Figure 1. (a) The obtained LSF from a single-pixel $50 \mathrm{~nm}$ FP LS SHIBL experiment. The insets show the LSF equation and an example of single-pixel $50 \mathrm{~nm}$ FP LS pattern with a He-ion line dose of 6.2 ions/nm in CAR. The green (blue) dots indicate our SHIBL experimental data for $40 \mathrm{~nm}(60 \mathrm{~nm})$ FP LS pattern. (b) The calculated PSF using Abel inversion of LSF. The blue dots indicate our SHIBL experimental data for a $40 \mathrm{~nm}$ FP CH pattern in CAR. The insets show the PSF equation and an example of single-pixel $40 \mathrm{~nm}$ FP CH pattern with a He-ion dose of 150 ions per contact hole in CAR. The CD-SEM data were translated into OCD data using first order correlation functions obtained from a CD-SEM vs OCD benchmark on EUV CAR resist metrology conducted by ASML (Veldhoven). ${ }^{13}$ 


\section{METHODOLOGY AND RESULTS}

\subsection{Modeling of single-pixel dose delivery with SHIBL in CAR}

Single-pixel exposure is modeled by converting a pixel dose map into a resist activation map. For instance, Figure 2(a) shows the pixel intensity dose map for an array of ten $500 \mathrm{~nm}$-long LS pattern at $50 \mathrm{~nm}$ FP, where each line is constructed from 250 single-pixel points with a pixel size of $2 \mathrm{~nm}$ (Figure 2(a) inset). Here, all pixels should receive the same dose. However, the actual dose delivered to each pixel depends on the Poisson distribution of the ion shot noise. The resultant pixel-intensity ion dose map is convolved with the PSF to obtain the resist activation map. Figure 2(b) shows the calculated resist activation $A(X, Y)$ map for the pattern of Figure 2(a) at a $\mathrm{He}^{+}$line dose of $5.5 \mathrm{ions} / \mathrm{nm}$. The activation $A$ is expressed in effective number of ions per unit of area. Note that the black regions in the resist activation map correspond to the exposed trenches and the lines are formed between these regions. Also, the blue-line indicates resist activation which corresponds to the dose-to-clear $D_{c}$ (i.e. $0.085 \mathrm{ions} / \mathrm{nm}^{2}$ ). ${ }^{6}$ Note that the dose-to-clear is defined as the threshold dose above which we assume the resist to fully dissolve after development. The metrology values (e.g. LWR, CD, LCDU) are evaluated based on the feature edge positions in the calculated resist activation map at the doseto-clear.

In the case of single-pixel exposure, target pixels are often considerably overexposed to achieve a desired $\mathrm{CD}$, unless the elements of the desired pattern have same size as the PSF. Furthermore, the developed resist patterns suffer from lower pattern fidelity (i.e. LCDU, LWR) with increasing dose. That is, in single-pixel exposures pattern fidelity is hampered by collective impact of several factors. First, most of the ion energy is deposited in the middle of the pattern and the pattern edges receive considerably lower effective dose. Second, the PSF tails define the pattern edges (which have smaller gradients than the central region of the PSF). Therefore, edge positions are more susceptible to dose fluctuations, resulting in lower pattern uniformity. Third, the impact of ion shot noise is more dominant in the PSF tails where the deposited ion energy is low. Therefore, not all relevant resist properties can be evaluated accurately with single-pixel exposure method.

(a)

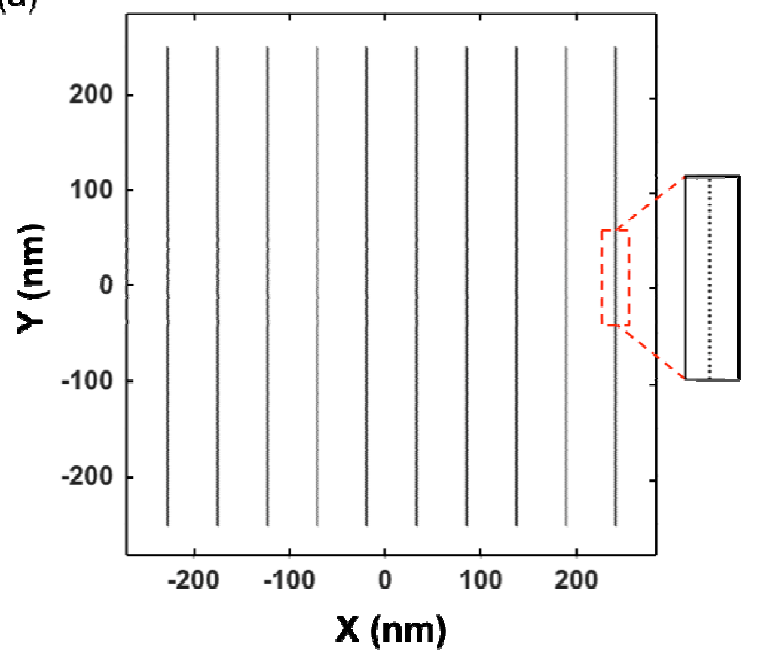

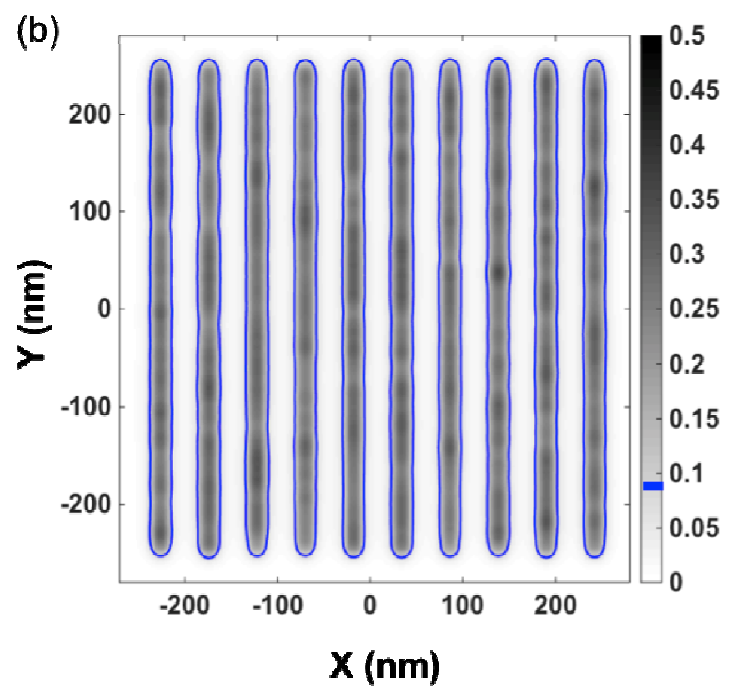

Figure 2. (a) Map for a single-pixel exposure of an array of ten $500 \mathrm{~nm}$-long LS pattern at $50 \mathrm{~nm}$-FP. The inset shows a segment of a line, which is constructed of single-pixels at $2 \mathrm{~nm}$ pitch. (b) The corresponding calculated resist activation map at a He-ion line dose of $5.5 \mathrm{ions} / \mathrm{nm}$. The blue-color-line on the gray scale bar indicates the dose-to-clear $D_{c}$. The bluecolor-lines indicate the pattern boundaries at the dose-to-clear $D_{c}$.

In order to validate our single-pixel dose delivery simulation model, we compared our calculations for line width and LWR of LS patterns and for CD and LCDU of CH patterns as function of He-dose with our experiments. To this end, we calculated an array of ten $2 \mu \mathrm{m}$-long $50 \mathrm{~nm}$-FP LS pattern for the same line dose range as the experiments (i.e. 2-11 ions $/ \mathrm{nm}$ ). Note that we discarded the two outer lines from each side of the array to calculate metrology parameters. It is also worth mentioning that due to the complexity of the resist activation calculations for a $2 \mu \mathrm{m}$-long LS pattern, the 
pattern was simulated four times as $500 \mathrm{~nm}$-long patterns and the generated results were then stitched together to construct the full pattern. Figure 3(a) compares the calculated resist activation maps to the experimental results for three different He-doses. Interestingly, the calculated resist activation maps exhibit decent resemblances of the experimental results. An array of LS pattern at 1:1 pitch was calculated to be achievable with a line dose of 7.3 ions $/ \mathrm{nm}$, which is slightly higher than that found in the experiments (i.e. 6.2 ions $/ \mathrm{nm}$ ). ${ }^{6}$ Also, the calculated LWR at $1: 1$ pitch is comparable to that of the experiment.
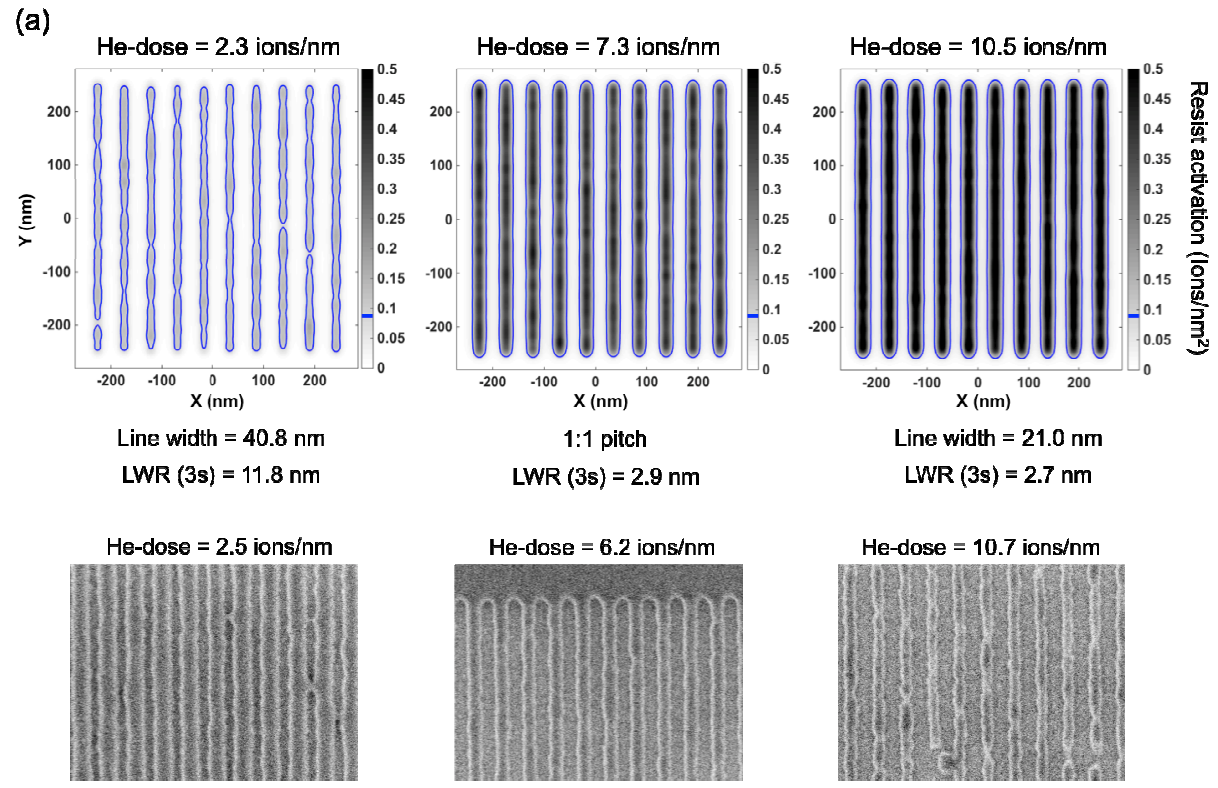

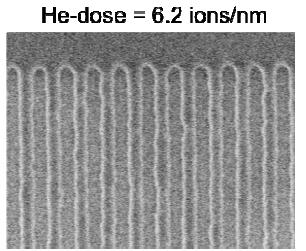

$1: 1$ pitch $\operatorname{LWR}(3 \mathrm{~s})=3.9 \mathrm{~nm}$

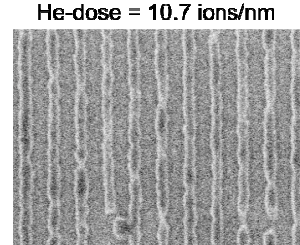

Line width $=20.1 \mathrm{~nm}$ $\operatorname{LWR}(3 \mathrm{~s})=6 \mathrm{~nm}$ $\operatorname{LWR}(3 \mathrm{~s})=4.6 \mathrm{~nm}$
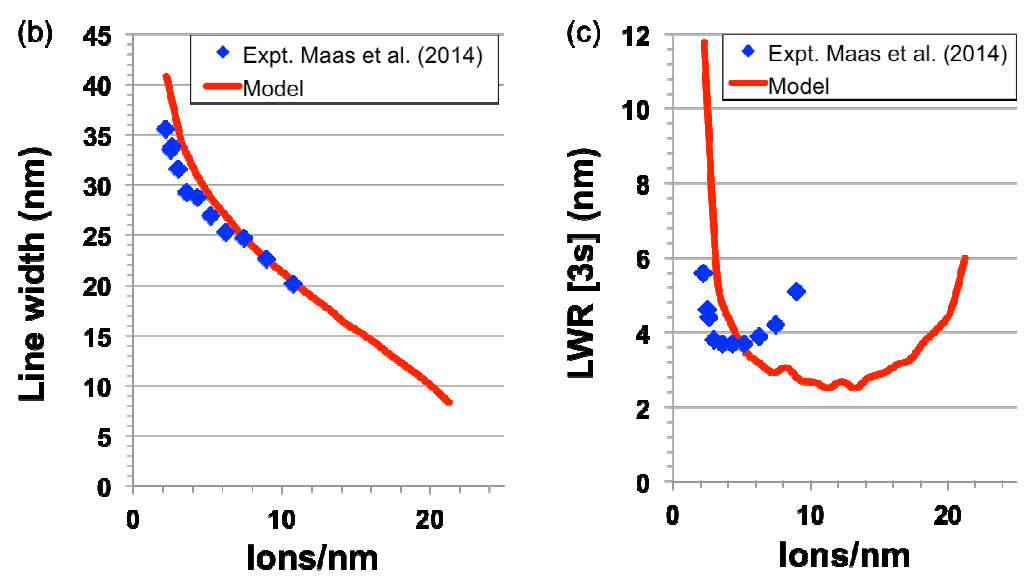

Figure 3. (a) Comparison between the calculated resist activation maps and the experimental results (SEM images) for different He-doses for a $50 \mathrm{~nm}$-full-pitch LS pattern. The blue-color-line on the gray scale bar indicates the dose-to-clear. Decent resemblance between each calculated resist activation map and its corresponding experimental result can be seen. (b) Comparison between the experiments and calculations for line width as a function of the He-dose, where a good agreement is obtained. (c) Comparison between the experiments and calculations for LWR as a function of the He-dose. The discrepancy is mainly attributed to instability of the ion source, inaccurate PSF estimation and not optimized experimental conditions.

The line width as a function of He-dose is illustrated in Figure 3(b). It is evident that the calculated line width values exhibit a good agreement with the experiments, and so validating our model for single-pixel SHIBL. Furthermore, the calculated LWR curve follows a trend similar to the experiments (Figure 3(c)). For He-ion line dose $\leq 6.3$ ions $/ \mathrm{nm}$ in the calculations (below 3 ions/nm for the experiments) the LWR is due to the ion shot noise. The second increase in the 
LWR at high He-ion doses is attributed to the contribution of the neighboring lines. Strangely, for He-doses below 4 ions/nm, our model estimates larger LWR values compared to the experiments. At the moment, we attribute this discrepancy to instability of the ion source due to contamination in the He gas at the moment of the experiments, ${ }^{13}$ inaccurate PSF estimation and not optimized experimental conditions (e.g. resist development) ${ }^{6}$.

For the contact hole pattern $(\mathrm{CH})$ at $40 \mathrm{~nm}-\mathrm{FP}$, we simulated an array of $10 \times 10$ to investigate the behavior of $\mathrm{CD}$ and LCDU as a function of He-ion dose (see Figure 4(a)). We then considered an inner array of $8 \times 8 \mathrm{CH}$ (the region inside the red-color-dashed line in Figure 4(a)) to perform metrology calculations. Figure 4(b) shows CD and LCDU as a function of the ion dose for the experiments and the simulations. In both cases, the CD increases with the ion dose. However, our model exhibits a quicker CD increase with the ion dose. On the other hand, the calculated LCDU is much lower than that observed in the experiments. For He-dose range of 80-150 ions per $\mathrm{CH}$, the calculated SHIBL LCDU is comparable to EUVL experiments (i.e. $2.9 \mathrm{~nm}$ ). ${ }^{6}$ For doses $\geq 150$ ions per $\mathrm{CH}$, the calculated LCDU values are on average $\sim 1 \mathrm{~nm}$ less than the obtained values for EUVL experiments. ${ }^{6}$ Therefore, the model suggests that our SHIBL experiments were not only limited by the ion shot noise. So by optimizing the experimental conditions ${ }^{6}$ better results could be achievable.
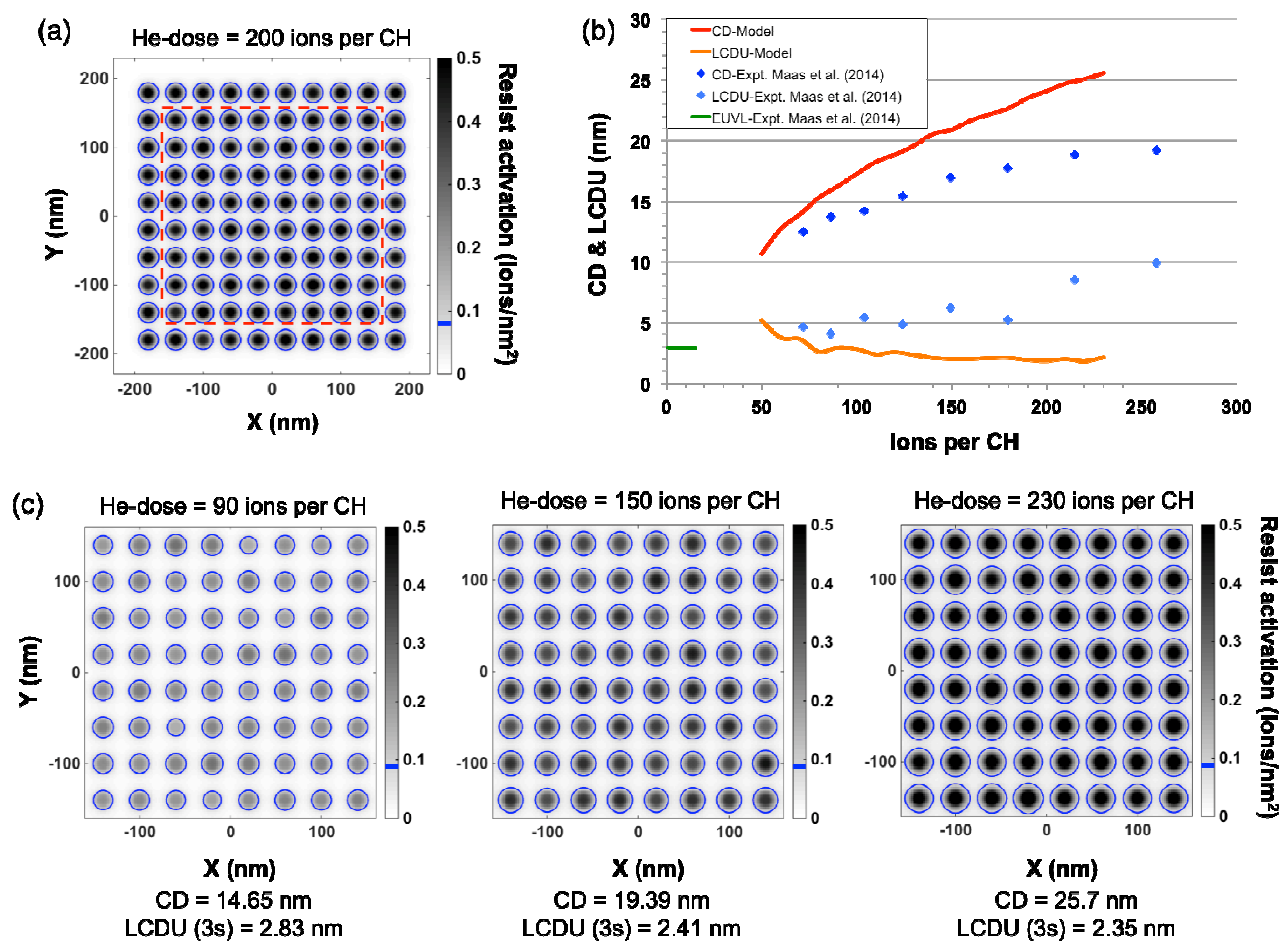

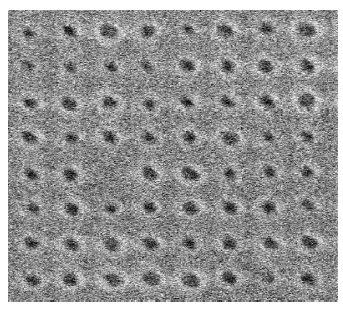

$\mathrm{CD}=13.7 \mathrm{~nm}$ $\operatorname{LCDU}(3 \mathrm{~s})=4.1 \mathrm{~nm}$

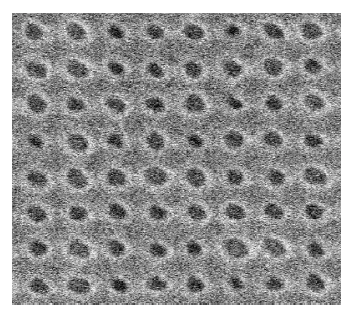

$\mathrm{CD}=17 \mathrm{~nm}$ $\operatorname{LCDU}(3 \mathrm{~s})=6.2 \mathrm{~nm}$

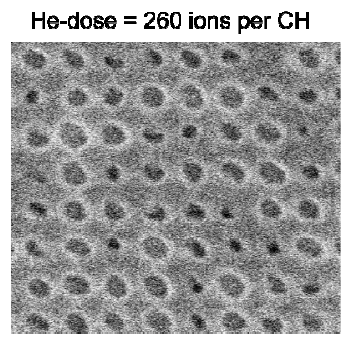

$C D=19.2 \mathrm{~nm}$

$\operatorname{LCDU}(3 \mathrm{~s})=9.9 \mathrm{~nm}$

Figure 4. (a) The calculated array of a $10 \times 10 \mathrm{CH}$ pattern where an inner array of $8 \times 8$ (within the red-dashed-square) is chosen to evaluate the results. The blue-line on the gray scale bar indicates the dose-to-clear. (b) Comparison between the calculated and CD-SEM metrology results for CD and LCDU. (c) Comparison between the calculated resist activation maps and SEM images of the experiments for the same He-dose. 
We attribute the large discrepancy between our model and experiments for $\mathrm{CH}$ pattern to instability of the ion source during our experiments and, to some extent, to inaccuracy of our PSF. Recently Rahman et al. reported on the beam emission intensity fluctuations in a Zeiss Orion helium ion microscope due to contaminants in the ion source region. ${ }^{14}$ Therefore, our experiments reported in Ref. 6 could have been affected by He-ion emission fluctuations for millisecond time intervals, and so affecting the delivered pixel dose at every dwell time. ${ }^{14}$ Therefore, the ion beam current fluctuations resulted in larger LCDU values. ${ }^{6}$ This can also be readily observed by comparing the calculated resist activation maps with the experimental results for the $\mathrm{CH}$ pattern in Figure 4(c). However, for LS patterns we scanned the beam over the desired pattern multiple times ("Repeat") to achieve the required dose. Therefore, each beam scan time was much shorter than the ones employed for the $\mathrm{CH}$ patterns, and so resulting in averaging of the beam current fluctuations over the entire pattern.

\subsection{Modeling of optimized-pixel-dose delivery with SHIBL in CAR}

We propose an optimized-pixel-dose delivery SHIBL to improve pattern fidelity to that obtained in single-pixel exposures. In this case, dose optimization offers two advantages over single-pixel exposures. First, dose optimization allows for a higher contrast around the dose-to-clear, and so improving the exposure latitude (EL). Second, the central area of large features can be realized with a significantly lower dose, thus improving throughput and avoiding pattern ablation. In the optimized-pixel-dose exposure we seek to minimize the discrepancy between a target dose profile and the realized dose profile by allowing all pixels (inside and outside of the feature) to contribute in a different way (i.e. with a different dose). Dose optimization is advantageous to single-pixel exposure when the feature size is at least about twice the width of the PSF.

The improved contrast for the dose-optimized exposure is due to the fact that the feature's edge (which after the dose map optimization is at the dose-to-clear) is exposed at the steepest part of the PSF. However, as previously mentioned, in single-pixel exposure the slope of the feature's dose profile around the dose-to-clear is progressively defined by the PSF tails with increasing the exposure margin (EM). In the optimized-pixel-dose exposure mode, the pixels far from the feature's edge do not contribute to the edge positions. Consequently, the required dose in the center of the feature is just above the dose-to-clear. However, in the single-pixel exposure mode, every exposed pixel also contributes to defining the feature's edge positions.

Our optimized-pixel-dose calculations are as follows. First, from a desired binary pattern (Figure 5(a)) we construct a target dose profile (Figure 5(b)). The target dose profile is calculated according to the capabilities of the exposure tool (e.g. resolution, beam step size, scanning beam electronics, etc.) and the resist properties (i.e. dose-to-clear). It is worth mentioning that in the model we assume the resist is not removed below the dose-to-clear. The model then finds iteratively a solution to an optimization problem in which we seek to minimize the discrepancy between the target dose profile and the realized dose profile. The solution provides the optimal ion doses at each pixel to achieve the best pattern fidelity (Figure 5(c)). It is worth mentioning that our dose optimization model is similar to the approach reported by Brodie et al..

(a)

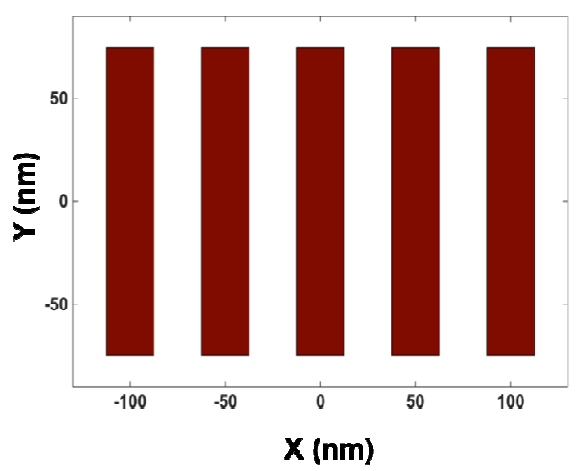

(b)

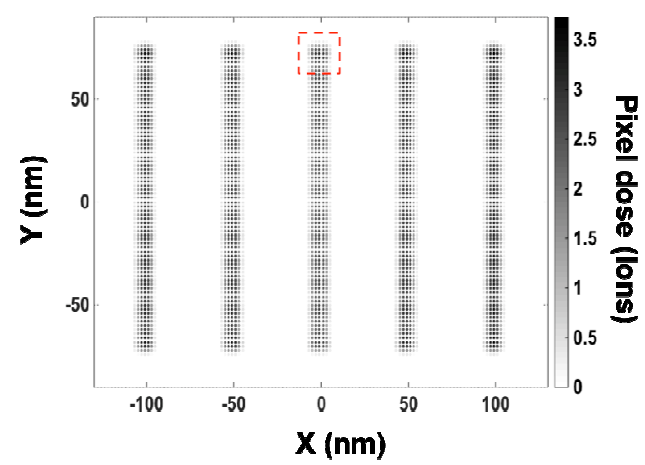

(c)

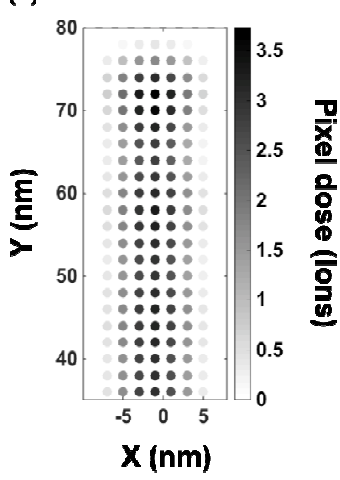

Figure 5. Graphical illustration of the calculation steps involved in our pixel dose optimization. (a) A binary map of a desired $50 \mathrm{~nm}$-FP LS pattern. (b) The calculated optimal ion dose map for the desired LS pattern based on the capabilities of the exposure tool and the resist properties. (c) The calculated optimal ion dose map for the region indicated by the red-colordashed rectangle in Figure 5(c). Here, each gray color represents the required number of ions for each pixel. 
Table 1 compares pattern fidelity of a $50 \mathrm{~nm}$-FP LS pattern for single-pixel and optimized-pixel-dose SHIBL exposure modes, based on our preliminary modeling results. Note that the exposure margin (EM) is defined as the ratio between the dose-to-size and the dose-to-clear and the exposure latitude (EL) is evaluated at $10 \%$ deviation from the target line width. Furthermore, Figure 6 shows the calculated resist activation maps for a $50 \mathrm{~nm}$-FP LS pattern for the EM values in Table 1. The images clearly show improvement in the LWR with increasing EM. For EM $\geq 4$, our model suggests that the optimized-pixel-dose exposures offer better LWR and comparable EL to that calculated for single-pixel exposures. The relatively large EL of single-pixel is due to the fact that if the desired feature size is larger than FWHM of the PSF (FWHM $=18.6 \mathrm{~nm}$ in our case), the tails of the PSF define the pattern edges. Consequently, a very large overexposure is required to achieve such feature sizes in single-pixel exposures. In contrast, in optimized-pixel-dose exposure a lower line dose (LD) is required to resolve the desired LS pattern (at a higher throughput) which at the same time exhibits lower LWR values (see Table 1). This is due to the fact that the pattern edges are no longer defined by the PSF tails, where the ion shot noise is dominating the exposure. Therefore, optimized-pixel-dose exposures can improve EL and pattern uniformity for feature sizes larger than the FWHM of the PSF. We also calculated that the impact of the ion shot noise on LWR ranges between 1.6-4.5 nm. Interestingly, the lower threshold is less than that of observed in EUVL experiments. ${ }^{6}$ However, further experiments and modeling data are required to truly investigate the ion shot noise impact and pattern fidelity in an optimized-pixel-dose SHIBL.

(a)

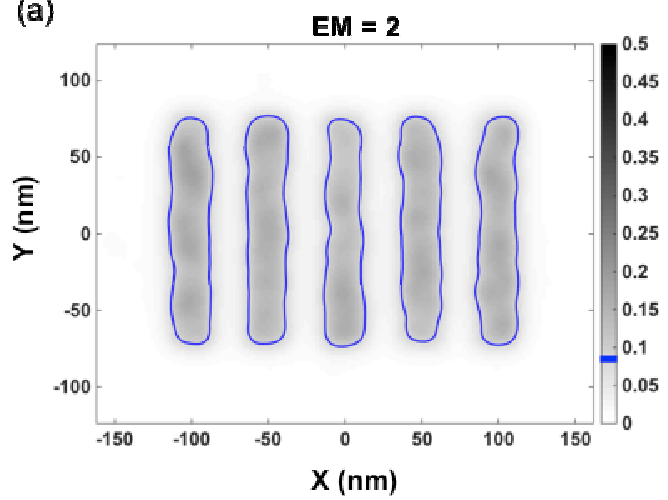

(c)

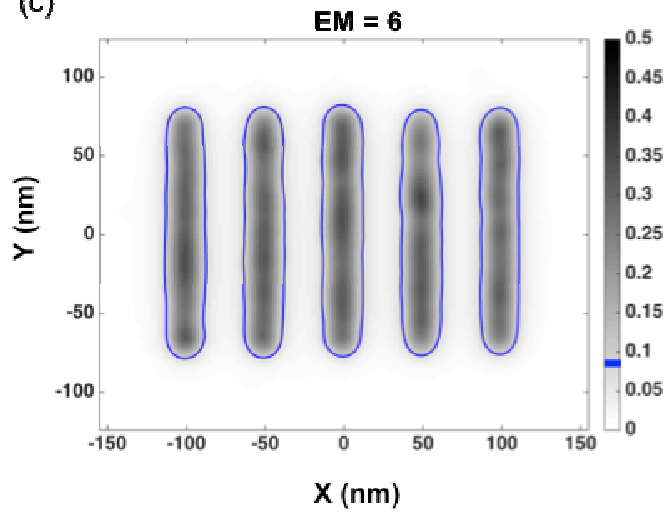

(b)

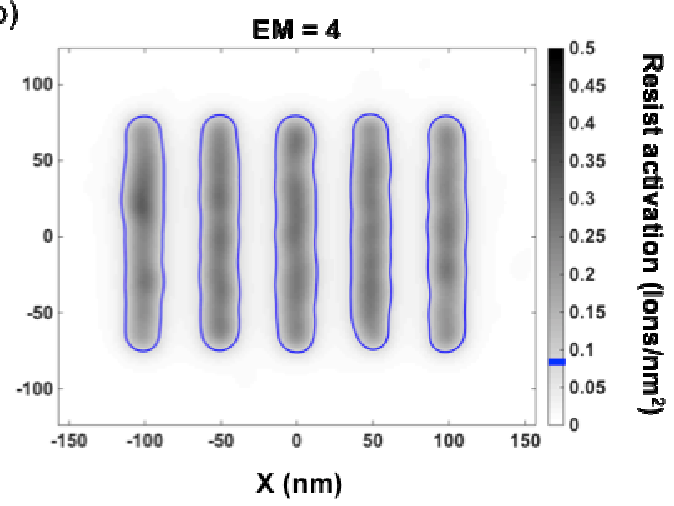

(d)

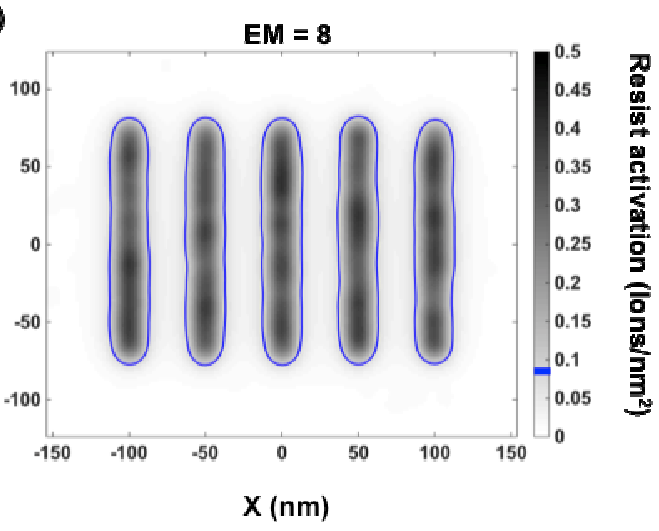

Figure 6. The calculated resist activation maps for optimized-pixel-dose exposure of a LS pattern at $50 \mathrm{~nm}$ FP for (a) EM = 2, (b) $\mathrm{EM}=4$, (c) $\mathrm{EM}=6$ and (d) $\mathrm{EM}=8$. The images clearly show the improvement in LWR with increasing EM. The blue-line on the gray scale bar indicates the dose-to-clear. 
Table 1. Preliminary comparison of EL, LWR and LD between single-pixel and optimized-pixel-dose SHIBL.

\begin{tabular}{|c|c|c|c|c|}
\cline { 2 - 5 } \multicolumn{1}{c|}{} & \multicolumn{4}{c|}{ Line-and-space at 50 nm FP } \\
\hline Exposure Mode & EM & EL & LWR (3s) & LD (ions/nm) \\
\hline Single-pixel & - & $45 \%$ & 2.9 & 7.3 \\
\hline \multirow{4}{*}{ Optimized-pixel-Dose } & 2 & $25 \%$ & 4.5 & 3.9 \\
\cline { 2 - 5 } & 4 & $40 \%$ & 2.5 & 5.0 \\
\cline { 2 - 5 } & 6 & $40 \%$ & 2.0 & 5.6 \\
\cline { 2 - 5 } & 8 & $42 \%$ & 1.6 & 6.2 \\
\hline
\end{tabular}

\section{CONCLUSIONS}

This paper presents a heuristic model for scanning helium ion beam lithography (SHIBL) in a EUV CAR. A point-spread function was employed to account for all physical and chemical phenomena involved in the resist activation. The pointspread function was obtained using Abel inversion of the line-spread function (acquired from SHIBL experiments for single-pixel $50 \mathrm{~nm}$-full-pitch line-and-space pattern). Ion shot noise effects were modeled with Poisson statistics. We discussed single-pixel and optimized-pixel-dose exposure modes as two possible approaches for SHIBL. We proposed the optimized-pixel-dose exposure mode to improve exposure margin, exposure latitude and line-width roughness in SHIBL. Our model showed a good agreement with the single-pixel SHIBL experiments for determining line width variations as a function of dose for a desired line-and-space pattern. The observed discrepancies between our modeling results and the experiments for line-width roughness, critical dimension and local critical dimension uniformity are attributed to inaccuracy of our estimate for the point-spread function, which in turn originates from experimental uncertainties. Therefore, better experimental data are required to improve the estimate of the point-spread function shape. In addition, further modeling and experimental data are required to determine the extent of ion shot noise effect on pattern fidelity in SHIBL in EUV CAR. Our preliminary modeling results indicated that optimized-pixel-dose SHIBL can offer better exposure latitude and line-width roughness in line-and-space patterns than single-pixel SHIBL. Therefore, optimized-pixel-dose SHIBL could pave the way towards employing SHIBL as a promising and a costeffective method for pre-screening EUV resists prior to their final performance evaluation in an EUV scanner.

\section{ACKNOWLEDGEMENTS}

We would like to acknowledge the Dutch Technology Foundation STW, which is part of the Netherlands Organization for Scientific Research (NWO), and the Ministry of Economic Affairs for funding this research. We would like to extend our gratitude to Timon Fliervoet, Sander Wuister, Rik Hoefnagels, Gijsbert Rispens, Vidya Vaenkatesan and Jeroen Meessen (ASML, Veldhoven, The Netherlands) for their contributions, advice and support. We would also like to thank Jochem P. B. Janssen (TNO, Delft, The Netherlands) for project management and, last but not least, Norbert Koster (TNO, Delft, The Netherlands) for presenting this paper at the conference.

\section{REFERENCES}

[1] Sidorkin, V., van Veldhoven, E., van der Drift, E., Alkemade, P., Salemink, H., Maas, D., "Sub-10-nm nanolithography with a scanning helium beam,” J. Vac. Sci. Technol. B 27(4), L18 (2009).

[2] Kubena, R. L., “A low magnification focused ion beam system with $8 \mathrm{~nm}$ spot size,” J. Vac. Sci. Technol. B Microelectron. Nanom. Struct. 9(6), 3079 (1991). 
[3] Winston, D., Cord, B. M., Ming, B., Bell, D. C., DiNatale, W. F., Stern, L. a., Vladar, a. E., Postek, M. T., Mondol, M. K., et al., "Scanning-helium-ion-beam lithography with hydrogen silsesquioxane resist," J. Vac. Sci. Technol. B 27(6), 2702 (2009).

[4] Melngailis, J., "Focused ion beam lithography," Nucl. Inst. Methods Phys. Res. B, 80-81(2), 1271-1280 (1993).

[5] Gamo, K., "Focused ion beam lithography,” Nucl. Inst. Methods Phys. Res. B 65, 40-49 (1992).

[6] Maas, D., van Veldhoven, E., van Langen-Suurling, A., Alkemade, P. F. A., Wuister, S., Hoefnagels, R., Verspaget, C., Meessen, J., Fliervoet, T., "Evaluation of EUV resist performance below 20nm CD using helium ion lithography," SPIE Adv. Lithogr., $90482 Z$ (2014).

[7] Wallow, T., Higgins, C., Brainard, R., Petrillo, K., Montgomery, W., Koay, C.-S., Denbeaux, G., Wood, O., Wei, Y., "Evaluation of EUV resist materials for use at the 32 nm half-pitch node," SPIE Adv. Lithogr., 692169211F (2008).

[8] Okoroanyanwu, U., "Chemistry and Lithography”, 861, Wiley, SPIE press (2011).

[9] Hawryluk, R. J., Smith, H. I., Soares, A., Hawryluk, A. M., "Energy dissipation in a thin polymer film by electron beam scattering: Experiment,” J. Appl. Phys. 46(6), 2528 (1975).

[10] Winston, D., Manfrinato, V. R., Nicaise, S. M., Cheong, L. L., Duan, H., Ferranti, D., Marshman, J., McVey, S., Stern, L., et al., "Neon Ion Beam Lithography (NIBL).," Nano Lett. 11(10), 4343-4347 (2011).

[11] Bunday, B., Cepler, A., Cordes, A., Arceo, A., “CD-SEM metrology for sub-10nm width features,” SPIE Adv. Lithogr., 90500T (2014).

[12] Bunday, B., Germer, T. A., Vartanian, V., Cordes, A., Cepler, A., Settens, C., "Gaps analysis for CD metrology beyond the 22nm node," SPIE Adv. Lithogr., 86813B 29 (2013).

[13] Maas, D. J., Fliervoet, T., Herfst, R., van Veldhoven, E., Meessen, J., Vaenkatesan, V., Sadeghian, H., "Sub-50 nm metrology on EUV chemically ampliefied resist-A systematic assessment" (In preparation for Rev. Sci. Instr.).

[14] Rahman, F. F., Notte, J. A., Livengood, R. H., Tan, S., "Observation of synchronized atomic motions in the field ion microscope.," Ultramicroscopy 126, 10-18 (2013).

[15] Brodie, A., Kojima, S., McCord, M., Grella, L., Gubiotti, T., Bevis, C., "Preliminary investigation of shot noise, dose, and focus latitude for e-beam direct write," SPIE Adv. Lithogr., 868029 (2013). 